\title{
Sex hormone-binding globulin, androgens and mortality: the KORA-F4 cohort study
}

\author{
Florian Schederecker ${ }^{1}$, Alexander Cecil ${ }^{2}$, Cornelia Prehn ${ }^{2}$, Jana Nano ${ }^{1}$, Wolfgang Koenig ${ }^{3,4}$, Jerzy Adamski ${ }^{2,5,6}$, \\ Tanja Zeller ${ }^{7,8}$, Annette Peters ${ }^{1,9}$ and Barbara Thorand ${ }^{1}$ \\ ${ }^{1}$ Institute of Epidemiology, Helmholtz Zentrum München - German Research Center for Environmental Health (GmbH), Neuherberg, Germany \\ ${ }^{2}$ Molecular Endocrinology and Metabolism, Genome Analysis Center, Helmholtz Zentrum München, German Research Center for Environmental Health, \\ Neuherberg, Germany \\ ${ }^{3}$ Institute of Epidemiology and Medical Biometry, University of UIm, Ulm, Germany \\ ${ }^{4}$ Deutsches Herzzentrum München, Technische Universität München, DZHK (German Centre for Cardiovascular Research), Partner Site Munich Heart \\ Alliance, Munich, Germany \\ ${ }^{5}$ Lehrstuhl für Experimentelle Genetik, Technische Universität München, Freising-Weihenstephan, Germany \\ ${ }^{6}$ Department of Biochemistry, Yong Loo Lin School of Medicine, National University of Singapore, Singapore, Singapore \\ ${ }^{7}$ Department of General and Interventional Cardiology, University Heart Center Hamburg, Hamburg, Germany \\ ${ }^{8}$ German Center for Cardiovascular Research (DZHK), Partner Site Hamburg/Kiel/Lübeck, Germany \\ ${ }^{9}$ German Centre for Cardiovascular Research (DZHK), Partner Site Munich, Munich, Germany
}

Correspondence should be addressed to B Thorand: thorand@helmholtz-muenchen.de

\begin{abstract}
Objective: Sex hormone-binding globulin (SHBG) and androgens have been associated with mortality in women and men, but controversy still exists. Our objective was to investigate associations of SHBG and androgens with all-cause and cause-specific mortality in men and women.

Design: 1006 men and 709 peri- and postmenopausal women (age range: 45-82 years)

from the German population-based KORA F4 cohort study were followed-up for a median of 8.7 years.

Methods: SHBG was measured with an immunoassay, total testosterone (TT) and dihydrotestosterone (DHT) with mass-spectrometry in serum samples and we calculated free testosterone (CFT). To assess associations between SHBG and androgen levels and mortality, we calculated hazard ratios (HRs) with 95\% Cls using Cox proportional-hazards models.

Results: In the cohort, 128 men (12.7\%) and 70 women (9.9\%) died. In women, we observed positive associations of SHBG with all-cause (HR: 1.54, 95\% Cl: 1.16-2.04) and with other disease-related mortality (HR: $1.86,95 \% \mathrm{Cl}: 1.08-3.20)$ and for DHT with allcause mortality (HR: $1.32,95 \% \mathrm{Cl}: 1.00-1.73$ ). In men, we found a positive association of SHBG (HR: 1.24 95\% Cl: 1.00-1.54) and inverse associations of TT (HR: $0.87,95 \%$ Cl: $0.77-0.97$ ) and CFT (HR: $0.84,95 \% \mathrm{Cl}: 0.73-0.97$ ) with all-cause mortality. No other associations were found for cause-specific mortality.

Conclusions: Higher SHBG levels were associated with increased risk of all-cause mortality in men and women. Lower TT and CFT levels in men and higher DHT levels in women were associated with increased risk of all-cause mortality. Future, well-powered population-based studies should further investigate cause-specific mortality risk.
\end{abstract}




\section{Introduction}

SHBG and sex hormones (e.g. androgens) have been shown to have deleterious or protective health effects, in particular in advanced age, and may account for some of the sex differences observed in cardiometabolic diseases and cancers $(1,2,3)$. In women with hyperandrogenism, an increased prevalence of metabolic risk factors and risk of type 2 diabetes (T2D) and cardiovascular disease (CVD) has been observed $(4,5,6)$. In men, high testosterone levels are associated with a protective CVD risk profile and lower risk for T2D and colorectal cancer $(6,7,8)$. Therefore, changes in androgen levels in middle aged and elderly persons seem to play an important role in modifying cardiometabolic risk and the occurrence of other chronic diseases. Consequently, these changes may affect mortality and there may be divergent effects in women and men, since men and women have markedly different androgen levels with different sex-specific functions (9, 10). Sex hormone binding globulin (SHBG) is a binding protein with high binding affinity for dihydrotestosterone (DHT), testosterone and estradiol (E2), and SHBG regulates their biological availability in the circulation (11). SHBG has been shown to be positively associated with all-cause and CVD mortality in elderly men (12). However, most studies have shown no associations for middle-aged men as well as for pre- and postmenopausal women $(13,14,15,16,17)$. Controversial results on the association of androgens with mortality have also been reported: several systematic reviews and meta-analyses provide consistent evidence for an inverse association of total testosterone (TT) and calculated free testosterone (cFT) levels with all-cause mortality in men, but results are not so conclusive for CVD mortality $(18,19,20,21$, $22,23)$. In pre- and postmenopausal women, results are inconclusive: some studies found no associations of TT or cFT levels with all-cause and CVD mortality $(16,17,24)$, while others reported inverse (25) or positive associations (26) with all-cause and CVD mortality. DHT is a bioactive metabolite of TT and possesses a strong binding affinity for SHBG, that is even stronger than the binding affinity of testosterone for SHBG (27). In men, associations of high DHT levels with lower CVD mortality (28) or curvilinear associations with all-cause mortality $(28,29)$ have been reported, while other studies could not find associations of DHT with mortality $(30,31)$. In women, we could not identify studies addressing the association of DHT with mortality. Overall, the association of SHBG and androgens with mortality still appears to be inconclusive. Therefore, our objective was to investigate prospective association of SHBG and androgens with all-cause as well as cause- specific mortality, adjusting for cardiometabolic and lifestyle factors among men and women in a large population-based study from Germany. Since SHBG is the major carrier protein for testosterone and DHT in the circulation, we also investigated the interactions of SHBG with TT as well as DHT levels regarding mortality.

\section{Materials and methods}

\section{Study design, setting and participants}

The Cooperative Health Research in the Region of Augsburg (KORA) F4 (2006-2008) is a follow-up examination of the population-representative KORA S4 study (1999-2001). Participants were community dwelling individuals, randomly selected from population registries in the city of Augsburg (Germany) and two surrounding counties. The study design has been described in detail previously (32). All participants underwent standard physical and medical examinations at KORA F4, which are described in detail elsewhere (33). The studies were carried out in accordance with the Declaration of Helsinki, including written informed consent from all participants, and were approved by the ethics committee of the Bavarian Chamber of Physicians (Munich, Germany). The KORA F4 study included 3080 participants (1486 men and 1594 women) aged 32-82 years. We restricted our analyses to persons aged $\geq 45$ years, because of only two recorded deaths among persons $<45$ years. Additionally, we excluded participants with missing values for SHBG/androgen measurements, missing values in at least one of the cofounding covariates, persons treated with exogenous sexual hormones (anti-estrogens, androgens, anti-androgens, enzyme-inhibitors, gonadotropin-releasing hormone analogs and anabolic steroids). Further, we excluded women with current pregnancy, women with self-reported bilateral oophorectomy or women with hysterectomy (if information on oophorectomy was missing) and premenopausal women. The exclusions are presented in a flow diagram in Supplementary Fig. 1 (see section on supplementary materials and methods given at the end of this article). The final sample for the analysis consisted of 1006 male and 709 female participants aged 45 to 82 years.

\section{Measurement of biomarkers}

At baseline, serum samples of the KORA F4 participants were collected and stored at $-80^{\circ} \mathrm{C}$ until analysis of 
SHBG and androgens. Measurements of SHBG in serum were performed using the ARCHITECT SHBG assay, a chemiluminescent microparticle immunoassay (CMIA) for absolute quantification of SHBG (Abbott Diagnostics) (Supplementary Materials and methods 1). E2, TT and DHT concentrations were quantified in serum with the AbsoluteIDQ ${ }^{\mathrm{TM}}$ Stero17 Kit (BIOCRATES Life Sciences AG, Innsbruck, Austria) using high performance liquid chromatography-tandem mass spectrometer (HPLC-MS/MS) (Supplementary Materials and methods 2). The LC-MS assays produced two batches of E2, DHT and TT measurements and therefore cross-calibration was performed for E2, TT and DHT measurements (Supplementary Materials and methods 3). FT was calculated (cFT) using the established formula by Vermeulen et al. (34). The calculation depends on TT, SHBG and albumin concentrations and the use of the equilibrium dissociation constants for the binding of SHBG and albumin with testosterone (35).

\section{Measurement of outcomes}

All participants of the KORA F4 study were followed-up for all-cause and cause-specific (CVD-, cancer- and other disease-related) mortality using death certificates, coded according to the International Classification of Diseases (originally ICD-9, translated to ICD-10). CVD-related mortality consists of diseases of the circulatory system (ICD-9 codes 390 - 459, ICD-10 codes I00-I99) and sudden death with unknown cause (ICD-9 code 798, ICD-10 code R99). Cancer-related mortality consists of neoplasms (ICD-9 codes 140-208, ICD-10 codes C00-C95). Other disease-related mortality consists of the remaining causes of death, for example, pneumonia (ICD-9 code 486, ICD-10 codes J18.8, J18.9), chronic bronchitis (ICD-9 code 491, ICD-10 Codes J41, J42, J44) and dementias (ICD-9 code 290, ICD-10 Codes F03.90, F05, F01.50, F01.51). Follow-up information on mortality was available until November 2016 (median follow-up time: 8.7 years, interquartile range (IQR): 8.2-9.1 years).

\section{Measurement of risk factors}

To control for confounding, a large panel of traditional and novel cardiovascular risk factors was assessed in the KORA F4 study. We used age in years at the baseline examination, systolic blood pressure in $\mathrm{mmHg}$, LDL cholesterol concentrations in $\mathrm{mmol} / \mathrm{L}$, use of lipid lowering medication (no/yes), use of antihypertensive medication (no/yes) and smoking (current/former/never).
Further, we assessed BMI $\left(\mathrm{kg} / \mathrm{m}^{2}\right)$, alcohol consumption (categorical) with different cutoffs for men (1, no alcohol; 2, alcohol consumption $>0$ to $<40 \mathrm{~g} /$ day; 3 , alcohol consumption $\geq 40 \mathrm{~g} /$ day) and women (1, no alcohol; 2 , alcohol consumption $>0$ to $<20 \mathrm{~g} /$ day; 3 , alcohol consumption $\geq 20 \mathrm{~g} /$ day), physical activity (inactive/active) and education in years (36). Further, we used estimated glomerular filtration rate (eGFR) which was calculated from serum creatinine (isotope dilution mass spectrometrycalibrated) with the 'Chronic Kidney Disease Epidemiology Collaboration' (CKD-EPI 2009) equation (37). High sensitivity C-reactive protein (CRP) levels were quantified in EDTA plasma with the CardioPhase hsCRP assay by means of particle-enhanced immunonephelometry using the BN II Systems from Siemens Healthcare Diagnostics. Self-reported or clinically diagnosed prevalent myocardial infarction (no/yes), prevalent stroke (no/yes) and prevalent cancer (no/yes) were also ascertained. To control for confounding by menopausal status, we characterized women as perimenopausal or postmenopausal. Women who reported a menstrual cycle within the last 12 months or women with a hysterectomy and $<60$ years of age were classified as perimenopausal. Women who reported having no menstrual cycle within the last 12 months or with a hysterectomy and $\geq 60$ years of age were classified as postmenopausal.

\section{Statistical analysis}

We reported categorical data as percentages and continuous data as means (S.D.), if they were normally distributed, or medians (IQR), if they were non-normally distributed. For analyzing differences between outcome groups (alive and dead), we used Wilcoxon rank-sum test for continuous variables and Chi-squared test for categorical variables. Cox proportional-hazards models were used to assess associations between SHBG/androgen levels and all-cause as well as CVD-, cancer- and other disease-related mortality. The highly skewed SHBG and androgen concentrations were log transformed to approximate normal distribution. Subsequently, we standardized the SHBG and androgen concentrations by dividing the log-transformed values by their sex-specific s.D. We calculated hazard ratios (HR) with 95\% CIs for all-cause and cause-specific mortality for a 1 s.D. increase on the log scale of the SHBG/androgen levels, adjusted for age and batch (model 1). For model 2 (multivariable adjusted model), we additionally adjusted for baseline CVD-risk and lifestyle factors: systolic blood pressure, LDL cholesterol concentrations, use of lipid lowering medication, use of antihypertensive medication,

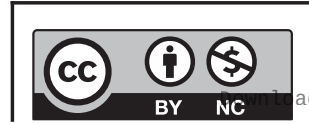

This work is licensed under a Creative Commons Attribution-NonCommercial 4.0 International License. ded from Bioscientifica.com at 04/26/2023 10:12:20AM 


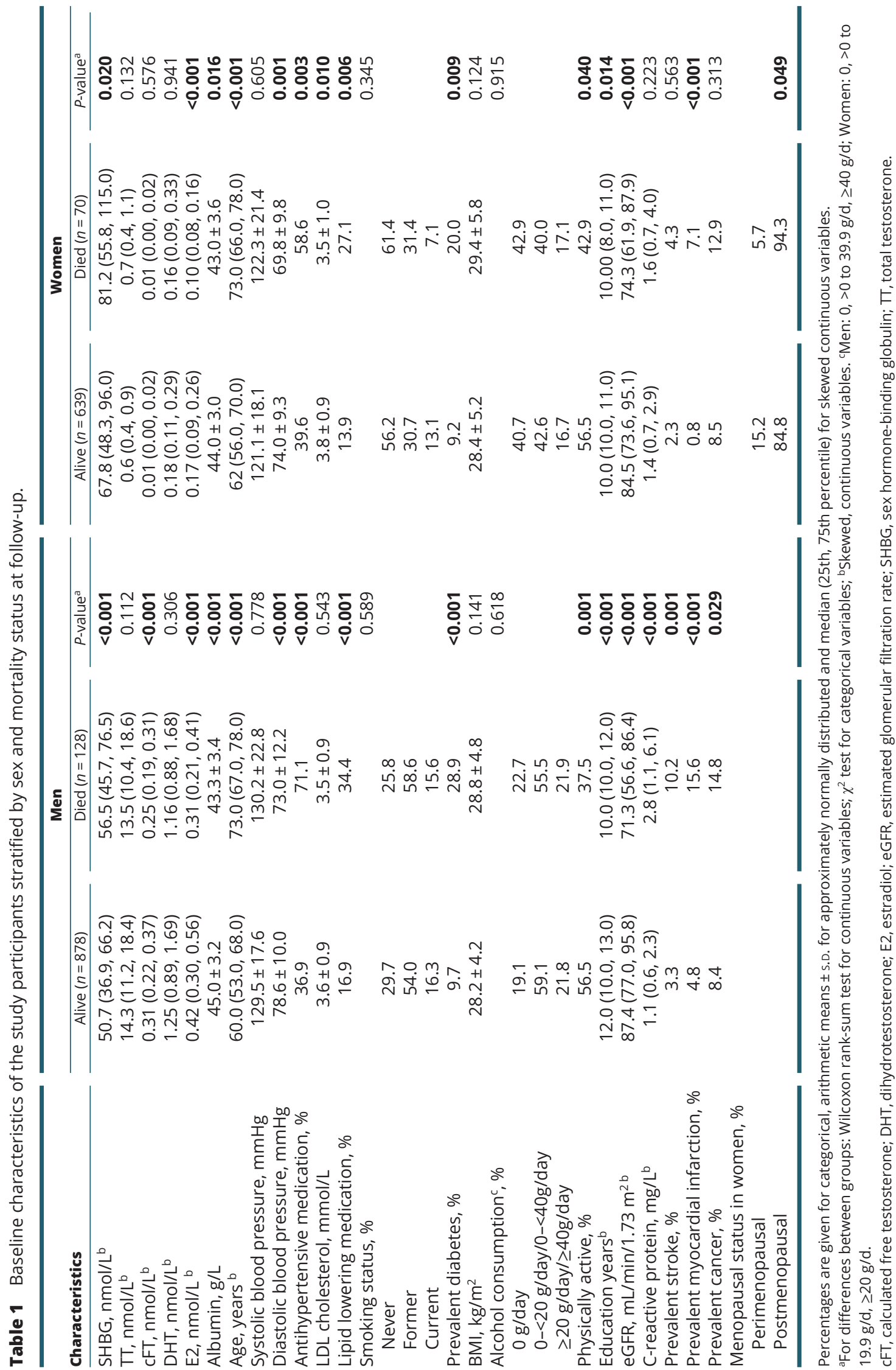


smoking and prevalent diabetes, BMI, categories of alcohol consumption, physical activity, education years, eGFR, CRP concentrations, prevalent myocardial infarction, prevalent stroke, prevalent cancer and menopausal status (perimenopausal/postmenopausal) in women. Since SHBG is the major carrier of testosterone and E2 in circulation (11), models assessing SHBG as exposure were also adjusted for TT and E2 in model 2. Due to their strong binding affinity to SHBG $(11,27)$, DHT and TT were also adjusted for SHBG levels. Additionally, we tested for interactions between SHBG and TT as well as between SHBG and DHT by inclusion of respective interaction terms in the Cox proportional-hazards models (model 3). We also tested interactions of the hormones with age (model 4). As a sensitivity analysis, we excluded deaths that occurred during the first 12 months to account for reverse causation due to serious morbidity. Additionally, we presented the doseresponse relationships of the hormones with mortality by using penalized smoothing splines (38). For this, we created plots with the splines of the SHBG/androgen concentrations against the hazard ratio with a $95 \%$ CI to check for possible non-linear forms of the associations (https://cran.r-project. org/package $=$ Greg). With a chi-squared test, we also checked quantitatively whether a nonlinear association was a significantly better fit than a linear association (38). The proportional hazards assumption was confirmed with Schoenfeld's tests for each single hormone and globally for the models (39). Test results with $P$-value $<0.05$ were considered statistically significant. We performed statistical analyses using R 3.5.1 (https://www.r-project.org/).

\section{Results}

Baseline characteristics of the study sample (1715 participants) are shown in Table 1 . In men, out of the
1006 participants, 128 (12.7\%) participants died (46.9\% CVD-related, $26.6 \%$ cancer-related and $26.6 \%$ other disease-related deaths) over the median follow-up period of 8.6 years (IQR: 8.2-9.1 years). In women, out of the 709 participants, 70 (9.9\%) participants died (35.7\% CVD-related, $37.1 \%$ cancer-related and $27.1 \%$ other disease-related deaths) over the median follow-up period of 8.7 years (IQR: 8.2-9.2 years). We observed substantial differences in the distributions and the range of SHBG and androgen levels between men and women (Supplementary Figs 2, 3, 4, 5 and 6), which supports the decision for a sex-specific analysis. In men, in the multivariable adjusted models (model 2), SHBG levels (HR per 1 s.D. log increase: $1.24,95 \%$ CI: $1.00-1.54)$ were significantly positively associated with all-cause mortality (Table 2 ). TT (HR: 0.87, 95\% CI: 0.77-0.98) and cFT (HR: 0.85, CI: 0.73-0.97) levels were significantly inversely associated with all-cause mortality. No association was found between DHT levels and all-cause mortality. We could not find interactions of SHBG with TT or DHT (model 3) and interactions of SHBG or the androgens with age regarding all-cause mortality in men (model 4). HRs tended to be similar to those of all-cause mortality for all cause-specific mortality outcomes. However, we could not observe any significant associations possibly due to smaller number of cases. After exclusion of persons, who died within the first 12 months of observation, the associations of SHBG, TT and cFT with all-cause mortality persisted (Supplementary Table 2). Figure 1 shows the penalized splines of the SHBG and androgens concentrations vs the hazard ratio with 95\% CIs regarding all-cause mortality in men. For SHBG and androgens, we can see graphically that a linear form is the adequate functional form. Chi-square tests confirmed this: a nonlinear form of these concentrations was not significantly better than a linear form, justifying our main analysis.

Table 2 Multivariable adjusted ${ }^{\mathrm{a}}$ associations of hormone levels with all-cause and cause-specific mortality: men $(n=1006)$.

\begin{tabular}{lc}
\hline All-cause mortality (events = 128) & SHBG \\
CVD - mortality (events = 60) & $\mathbf{1 . 2 4}(\mathbf{1 . 0 0 , 1 . 5 4 )}$ \\
Cancer - mortality (events $=34)$ & SHBG \\
& $1.23(0.88,1.72)$ \\
Other disease - mortality (events = 34) & SHBG \\
& $1.29(0.84,1.97)$ \\
& SHBG \\
& $1.32(0.89,1.96)$
\end{tabular}

TT
$\mathbf{0 . 8 7}(\mathbf{0 . 7 7}, \mathbf{0 . 9 8})$
TT
$0.90(0.70,1.17)$
TT
$0.85(0.69,1.04)$
$T T$
$0.87(0.71,1.05)$

$\mathrm{CFT}$
$\mathbf{0 . 8 5}(\mathbf{0 . 7 3}, \mathbf{0 . 9 7})$
$\mathrm{CFT}$
$0.85(0.65,1.12)$
$\mathrm{CFT}$
$0.84(0.67,1.06)$
$\mathrm{CFT}$
$0.84(0.65,1.09)$

\begin{tabular}{c}
\hline $\mathrm{DHT}$ \\
$0.89(0.77,1.03)$ \\
$\mathrm{DHT}$ \\
$0.93(0.70,1.24)$ \\
$\mathrm{DHT}$ \\
$0.90(0.71,1.13)$ \\
$\mathrm{DHT}$ \\
$0.88(0.67,1.15)$ \\
\hline
\end{tabular}

Data are reported as hazard ratios $(95 \% \mathrm{Cl})$ per 1 logarithmic s.D. increase with $P<0.05$ marked as bold; $n$, sample size. ${ }^{a}$ Adjusted for age, batch, systolic blood pressure, LDL cholesterol, use of lipid lowering medication, smoking status, use of antihypertensive medication, prevalent diabetes, BMI, education years, physical activity, alcohol consumption, estimated glomerular filtration rate, high-sensitivity C-reactive protein concentration, prevalent cancer, prevalent myocardial infarction and prevalent stroke. Additionally, SHBG is adjusted for TT and E2; DHT and TT are adjusted for SHBG.

cFT, calculated free testosterone; DHT, dihydrotestosterone; E2, estradiol; SHBG, sex hormone-binding globulin; TT, total testosterone.

https://ec.bioscientifica.com

https://doi.org/10.1530/EC-20-0080 (c) 2020 The authors Published by Bioscientifica Ltd

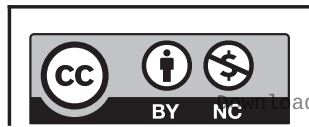

This work is licensed under a Creative Commons Attribution-NonCommercial 4.0 International License. ded from Bioscientifica.com at 04/26/2023 10:12:20AM 
SHBG

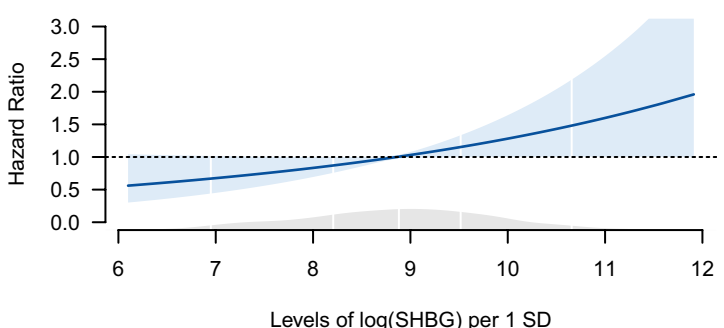

cFT

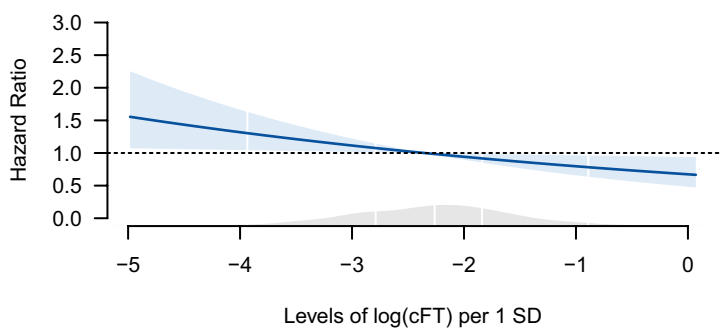

TT

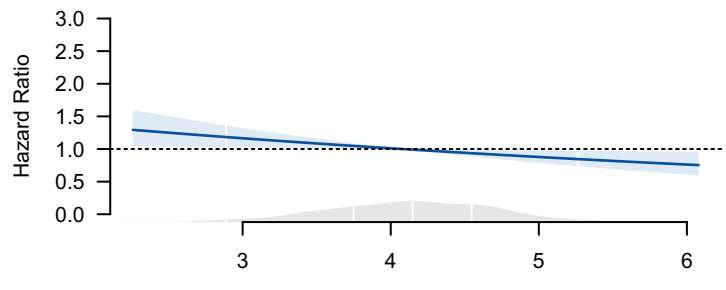

Levels of $\log (T T)$ per $1 \mathrm{SD}$

DHT

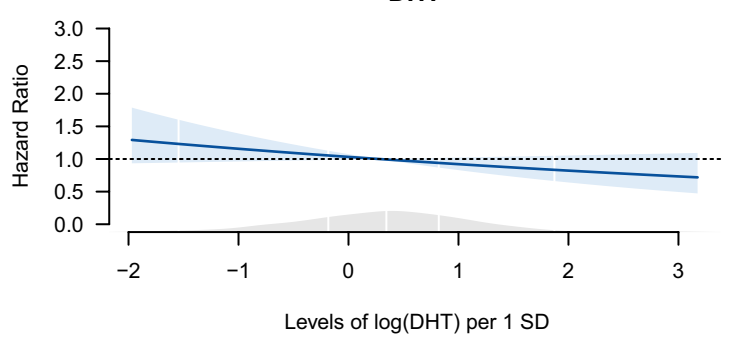

\section{Figure 1}

Splines of SHBG and androgen concentrations: all-cause mortality in men. SHBG, sex hormone-binding globulin; TT, total testosterone; cFT, calculated free testosterone; DHT, dihydrotestoterone; E2, Estradiol. Multivariable adjusted ${ }^{a}$ Cox proportional hazards models exploring hormone levels and associations with all-cause mortality. SHBG and androgens levels are entered into the model as penalized smoothing spline. White vertical lines represent $2.5 \%, 25 \%, 50 \%, 75 \%$ and $97.5 \%$ quantiles of SHBG/androgen levels. The blue shaded area denotes the $95 \% \mathrm{Cl}$ of the spline estimation. The

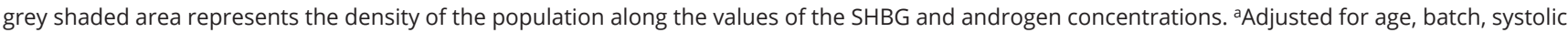
blood pressure, LDL cholesterol, use of lipid lowering medication, smoking status, use of antihypertensive medication, prevalent diabetes, body mass index, education years, physical activity, alcohol consumption, estimated glomerular filtration rate, high-sensitivity C-reactive protein concentration, prevalent cancer, prevalent myocardial infarction, prevalent stroke. Additionally, SHBG is adjusted for TT and E2; TT and DHT are adjusted for SHBG.

In women, in the multivariable adjusted models (model 2), SHBG (HR: 1.54, 95\% CI: 1.16-2.04) and DHT (HR: 1.32, CI: 1.00-1.73) levels were significantly positively associated with all-cause mortality (Table 3). In cause-specific mortality analyses, SHBG was also positively associated with other disease-related mortality (HR: 1.86, 95\% CI: 1.08-3.20). Androgen levels were not associated with cause-specific mortality. After exclusion of persons, who died within the first 12 months of observation, the association of SHBG with mortality persisted, but the association between DHT and mortality became insignificant (HR: 1.25, 95\% CI: 0.96-1.64) (Supplementary Table 2). Figure 2 shows the spline results for women. For SHBG and DHT levels, a more flexible functional form can be observed graphically, but a nonlinear form was not significantly better than a linear form according to chi-square tests, justifying our main analysis. A positive interaction of SHBG levels with TT levels $(P=0.027$, model 3) and a positive interaction of SHBG levels with age $(P=0.006$, model 4) regarding all-cause mortality was found in women. This means that, for increasing levels of TT, the association of SHBG levels with all-cause mortality also increased. Additionally, for increasing age, the association of SHBG levels with all-cause mortality also increased. Analysis stratified for tertiles of TT showed that SHBG levels were only significantly positively associated with all-cause mortality in the highest tertile of TT. Analysis stratified for 10-year age groups showed that SHBG levels were only significantly positively associated with all-cause mortality in the age group of 75-82 years. Using Schoenfeld's tests, we found no violation of the proportional hazards assumption for SHBG and androgen levels. Global tests for the overall models also showed no violation of the proportional hazards assumption.

\section{Discussion}

In this prospective cohort study of middle-aged and elderly men and women from the general population in Germany, SHBG was positively associated with all-cause mortality in both men and women, while androgens were differently associated with all-cause mortality in men and women. Increasing TT and cFT levels were associated with decreased risk of all-cause mortality in men, while increasing DHT levels were associated with increased risk of all-cause mortality in women.

Additionally, we observed positive interactions of SHBG with TT and SHBG with age in women for all-cause mortality. 
Table 3 Multivariable adjusted associations of hormone levels with all-cause and cause-specific mortality: women $(n=709)$.

All-cause mortality (events $=70)$
CVD - mortality (events $=25)$
Cancer - mortality (events $=26)$
Other disease - mortality (events $=19$ )

\begin{tabular}{c} 
SHBG \\
$\mathbf{1 . 5 4}(\mathbf{1 . 1 6 , 2 . 0 4 )}$ \\
SHBG \\
$1.18(0.72,1.91)$ \\
SHBG \\
$1.42(0.89,2.26)$ \\
SHBG \\
$\mathbf{1 . 8 6}(\mathbf{1 . 0 8 , 3 . 2 0})$ \\
\hline
\end{tabular}

\begin{tabular}{c}
$\pi T$ \\
$1.12(0.84,1.49)$ \\
$\pi T$ \\
$1.08(0.66,1.78)$ \\
$\pi$ \\
$1.06(0.70,1.60)$ \\
$\pi T$ \\
$1.11(0.64,1.92)$ \\
\hline
\end{tabular}

\begin{tabular}{c}
\hline $\mathrm{CFT}$ \\
$0.86(0.66,1.11)$ \\
$\mathrm{CFT}$ \\
$1.08(0.71,1.67)$ \\
$\mathrm{CFT}$ \\
$0.77(0.50,1.17)$ \\
$\mathrm{CFT}$ \\
$0.87(0.54,1.39)$ \\
\hline
\end{tabular}

\begin{tabular}{c} 
DHT \\
$\mathbf{1 . 3 2}(\mathbf{1 . 0 0}, \mathbf{1 . 7 3})$ \\
DHT \\
$1.53(0.93,2.52)$ \\
DHT \\
$1.63(0.95,2.78)$ \\
DHT \\
$0.98(0.67,1.43)$ \\
\hline
\end{tabular}

Data are reported as hazard ratios $(95 \% \mathrm{Cl})$ per 1 logarithmic s.D. increase with $P<0.05$ marked in bold; $\mathrm{n}$, sample size.

aAdjusted for age, batch, systolic blood pressure, LDL cholesterol, use of lipid lowering medication, smoking status, use of antihypertensive medication, prevalent diabetes, BMI, education years, physical activity, alcohol consumption, estimated glomerular filtration rate, menopausal status, high-sensitivity C-reactive protein concentration, prevalent cancer, prevalent myocardial infarction and prevalent stroke. SHBG is adjusted for TT and E2; DHT and TT are adjusted for SHBG.

cFT, calculated free testosterone; DHT, dihydrotestosterone; E2, estradiol; SHBG, sex hormone-binding globulin; TT, total testosterone.

\section{Androgens and mortality in men}

Our findings confirm the results from previous studies, which observed inverse associations between TT (14, $40,41,42)$ and cFT $(14,30,42,43)$ levels and all-cause mortality in men. Concerning the cause-specific analysis, the evidence in the literature so far has been inconclusive, reporting either inverse associations or no relation at all. Results of recent meta-analyses indicated low TT levels to be associated with increased CVD mortality $(19,22)$. In our study, we found no associations of TT and cFT with
CVD-, cancer and other disease-related mortality. Lower testosterone levels in men are associated with type 2 diabetes, hepatic steatosis, coronary artery disease as well as other diseases such as chronic obstructive pulmonary disease and chronic renal disease $(44,45,46)$. Thus, low androgen levels may be a marker of poor general health rather than an independent predictor of mortality (18, $19,21)$. However, the observed associations between low TT and cFT levels and all-cause mortality were adjusted for a number of chronic diseases (i.e. prevalent CVD, cancer, diabetes). Additionally, we excluded deaths that
SHBG

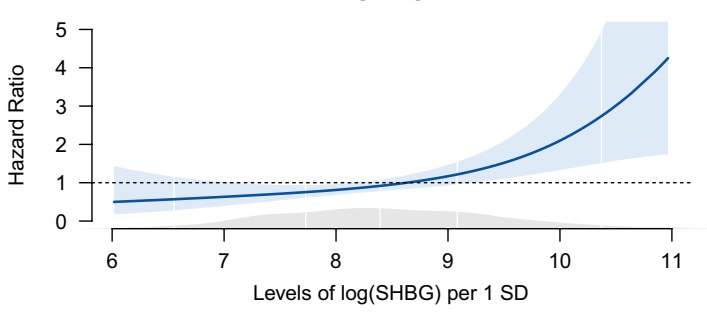

cFT

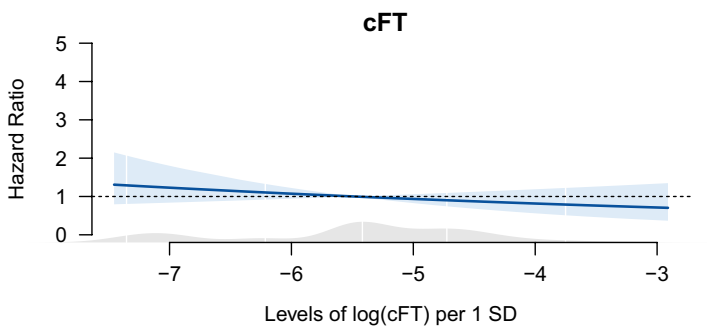

TT

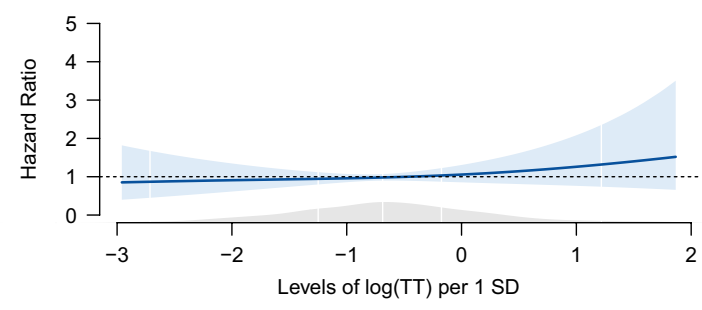

DHT

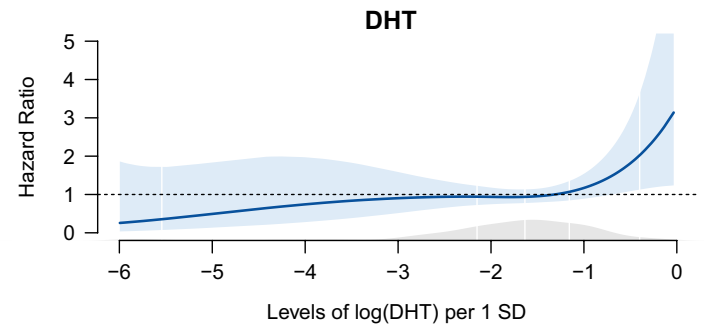

Figure 2

Splines of SHBG and androgens concentrations: all-cause mortality in women. SHBG, sex hormone-binding globulin; TT, total testosterone; cFT, calculated free testosterone; DHT, dihydrotestoterone; E2, estradiol. Multivariable adjusted ${ }^{a}$ Cox proportional hazards models exploring hormone levels and associations with all-cause mortality. SHBG and androgens levels are entered into the model as penalized smoothing spline. White vertical lines represent $2.5 \%, 25 \%, 50 \%, 75 \%$ and $97.5 \%$ quantiles of SHBG/androgen levels. The blue shaded area denotes the $95 \% \mathrm{Cl}$ of the spline estimation. The

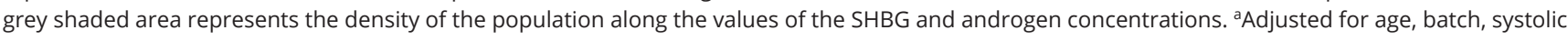
blood pressure, LDL cholesterol, use of lipid lowering medication, smoking status, use of antihypertensive medication, prevalent diabetes, body mass index, education years, physical activity, alcohol consumption, estimated glomerular filtration rate, high-sensitivity C-reactive protein concentration, menopausal status, prevalent cancer, prevalent myocardial infarction, prevalent stroke. Additionally, SHBG is adjusted for TT and E2; TT and DHT are adjusted for SHBG.

https://ec.bioscientifica.com

https://doi.org/10.1530/EC-20-0080 (c) 2020 The authors Published by Bioscientifica Ltd

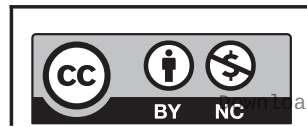

This work is licensed under a Creative Commons Attribution-NonCommercial 4.0 International License. ded from Bioscientifica.com at 04/26/2023 10:12:20AM 
occurred during the first 12 months of follow-up and the association with all-cause mortality remained significant for both TT and cFT (Supplementary Table 2). However, it is worth mentioning that results for cFT did not provide any additional information beyond those for TT levels. Despite these robust results, this does not rule out the possibility that low androgen levels are just markers of poor general health, since there are still unmeasured possible confounders including sleep disordered breathing and depression. When we further adjusted TT and cFT levels for E2, the associations with all-cause mortality became insignificant (Supplementary Table 1).

\section{Androgens and mortality in women}

In the present analysis, DHT levels were positively associated with all-cause mortality in women. We are not aware of any other study examining this relationship. DHT represents a potent androgen with deleterious effects on metabolic risk. However, its physiological significance is still poorly studied in women (47). The finding of a significant positive association between DHT and allcause mortality needs to be interpreted with high caution: Although it is in line with results for CVD- and cancermortality, the association with all-cause mortality is of borderline significance with rather wide CIs. Additionally, the cause-specific results are very instable, due to the very small number of cases. When we excluded deaths that occurred during the first year of follow-up, the association of DHT with all-cause mortality disappeared (HR: 1.25, 95\% CI: 0.96-1.64), hinting towards reverse causation (Supplementary Table 2). Our non-significant findings regarding TT and cFT levels and mortality are in line with some previous studies $(16,17,24)$, while other studies found inverse (25) or positive (26) associations. Given these contradictory results, further larger studies should elucidate these associations in women.

\section{SHBG and mortality}

Regarding SHBG levels, we observed positive associations with all-cause mortality, confirming previous results for elder men (12). However, the result is of borderline significance and needs to be interpreted with caution. Regarding cause-specific mortality, we observed no associations with SHBG levels. Another study of adult men (20-90 years, mean age: 40 years) from the general population observed a positive association of SHBG with CVD- mortality in the first 9 years and an inverse association in the second period from 9 to 18 years (48).
Thus, varying follow-up periods could affect results. In contrast to the few available studies that could not find an association between SHBG levels with all-cause or specific mortality among women $(15,16,17)$, we found a positive association with all-cause mortality. We also found a positive association of SHBG with other diseaserelated mortality in women, but this finding needs to be considered with caution due to limited number of events (19). Different hypotheses might explain the effect of SHBG on mortality:

1. Indirect effect of SHBG - via regulating testosterone action through its binding properties: the majority of circulating testosterone is bound to SHBG. Higher SHBG levels - by binding more testosterone - could limit the amount of bioactive testosterone leading to a reduction in tissue androgenisation. Our results provide some evidence in support for an indirect effect of SHBG, since cFT was inversely associated and SHBG levels were positively associated with mortality in men. Additionally, cFT tended to be inversely associated with mortality (although not significantly), whereas higher levels of SHBG were significantly associated with increased risk of mortality in women. The significant positive interaction of SHBG with TT regarding mortality in women adds up on evidence for an indirect effect. However, analysis stratified for tertiles of TT showed that SHBG levels were only significantly positively associated with all-cause mortality in the highest tertile of TT. Analysis stratified for 10-year age groups showed that SHBG levels were only significantly positively associated with all-cause mortality in the age group of $75-82$ years. Possible explanations for that include that SHBG increases markedly with weight loss and undernutrition (49, 50). Unfortunately, BMI (which used in our analysis) is a relatively crude measure of body composition. Additionally, higher levels of SHBG are associated with increased frailty and fracture $(11,51)$.

2. Direct effect of SHBG - as suggested by preclinical models (52): only unliganded, but not sex steroidbound SHBG binds to the SHBG receptor, suggesting that SHBG may have biological actions that are independent of sex steroids $(53,54)$. Our finding of a significant association of SHBG levels with allcause mortality even after adjustment for E2 and TT levels would support the direct link between SHBG and mortality. However, it is also possible that high SHBG levels just represent a marker of poor health. In line with this possibility, malnutrition and energy https://ec.bioscientifica.com https://doi.org/10.1530/EC-20-0080 (c) 2020 The authors Published by Bioscientifica Ltd
This work is licensed under a Creative Commons Attribution-NonCommercial 4.0 International License. ded from Bioscientifica.com at 04/26/2023 10:12:20AM 
deficits, in particular, among the elderly men and women can increase SHBG levels $(49,50)$. To account for that, we excluded deaths that occurred during the first 12 months of follow-up, but the independent association with all-cause mortality remained significant for SHBG in men and women. There are possible unmeasured confounders of potential importance in interpreting the SHBG results, which we were not able to include in the analysis. Information about liver disease would have been useful since, depending on type and stage, elevations of SHBG levels may occur; elevations of thyroid hormone may increase SHBG levels and hypothyroidism may lower SHBG levels and diabetes medications also may affect SHBG levels (11).

\section{Strengths and limitations}

Strengths of our study include the population-based longitudinal design, adjustment for a wide range of cardiometabolic and lifestyle risk factors and complete capture of death events via registry linkage. Additionally, androgens were measured optimally respecting diurnal variability (fasting samples obtained in the morning) and with state-of-the-art methodology. However, there are a few limitations worth mentioning. The results for women - especially for cause-specific mortality - are underpowered due to the small number of cases, resulting in wide CIs that do not exclude strong, direct or inverse associations. Even though there are insufficient outcome events for meaningful analysis of cause-specific mortality, the major findings of DHT and SHBG with respect to all-cause mortality are noteworthy. Although multiple measurements on the same day might be more meaningful, a single measurement of testosterone is considered reliable in the context of large-scale analyses (55). Additionally, the amplitude of diurnal variation of DHT and SHBG at all ages is either considerably smaller or not detectable (56). Limitations also include the estimation of FT, since FT levels were not measured directly. Therefore, the results should be interpreted carefully. The Vermeulen method has been reported to both overestimate and underestimate FT compared with laboratory measures (57), but direct measurement of FT was not available. However, cFT has been shown to be highly correlated with direct measurements (35). The LC-MS assays produced two batches of DHT and TT measurements and therefore were cross-calibrated. However, there was a very high correlation between batch 1 and 2 measurements and we performed well-established calibration methods (Supplementary Materials and methods 3).

\section{Conclusion}

In summary, this study shows that higher SHBG levels are associated with increased risk of all-cause mortality in middle-aged and older women and men, independent of known confounders. Additionally, higher DHT levels in women and lower TT and FT levels in men are associated with increased risk of all-cause mortality. These results should prompt further investigations of the role of SHBG and androgens in the health of middle-aged and older men and women. Additionally, future well-powered population-based studies should further investigate causespecific mortality risk.

\section{Supplementary materials}

This is linked to the online version of the paper at https://doi.org/10.1530/ EC-20-0080.

\section{Declaration of interest}

The authors declare that there is no conflict of interest that could be perceived as prejudicing the impartiality of the research reported.

\section{Funding}

This project was supported by the German Center of Cardiovascular Research (DZHK) by a research grants within the shared expertise programme (DZHK B 17-035 SE) and by funding from the Helmholtz Alliance 'Aging and Metabolic Programming, AMPro'. Tanja Zeller is supported by the German Center for Cardiovascular Research (DZHK 81Z1710101, FKZ 8120710102 and FKZ 81X2710160).

\section{References}

1 Bjornerem A, Straume B, Midtby M, Fønnebø V, Sundsfjord J, Svartberg J, Acharya G, Oian P \& Berntsen GK. Endogenous sex hormones in relation to age, sex, lifestyle factors, and chronic diseases in a general population: the Tromso Study. Journal of Clinical Endocrinology and Metabolism 200489 6039-6047. (https://doi. org/10.1210/jc.2004-0735)

2 Humphries KH, Izadnegahdar M, Sedlak T, Saw J, Johnston N, Schenck-Gustafsson K, Shah RU, Regitz-Zagrosek V, Grewal J, Vaccarino V, et al. Sex differences in cardiovascular disease - impact on care and outcomes. Frontiers in Neuroendocrinology 201746 46-70. (https://doi.org/10.1016/j.yfrne.2017.04.001)

3 Appelman Y, van Rijn BB, Ten Haaf ME, Boersma E \& Peters SA. Sex differences in cardiovascular risk factors and disease prevention. Atherosclerosis 2015241 211-218. (https://doi.org/10.1016/j. atherosclerosis.2015.01.027)

4 Finch CE. The menopause and aging, a comparative perspective. Journal of Steroid Biochemistry and Molecular Biology 2014142 132-141. (https://doi.org/10.1016/j.jsbmb.2013.03.010)
This work is licensed under a Creative Commons Attribution-NonCommercial 4.0 International License. ded from Bioscientifica com at 04/26/2023 10:12:20AM 
5 Dokras A. Cardiovascular disease risk in women with PCOS. Steroids 201378 773-776. (https://doi.org/10.1016/j.steroids.2013.04.009)

6 Ding EL, Song Y, Malik VS \& Liu S. Sex differences of endogenous sex hormones and risk of type 2 diabetes: a systematic review and meta-analysis. JAMA 2006295 1288-1299. (https://doi.org/10.1001/ jama.295.11.1288)

7 Ding EL, Song Y, Manson JE, Hunter DJ, Lee CC, Rifai N, Buring JE, Gaziano JM \& Liu S. Sex hormone-binding globulin and risk of type 2 diabetes in women and men. New England Journal of Medicine 2009 361 1152-1163. (https://doi.org/10.1056/NEJMoa0804381)

8 Lin JH, Zhang SM, Rexrode KM, Manson JE, Chan AT, Wu K, Tworoger SS, Hankinson SE, Fuchs C, Gaziano JM, et al. Association between sex hormones and colorectal cancer risk in men and women. Clinical Gastroenterology and Hepatology 201311 419.e1-424 e1. (https://doi.org/10.1016/j.cgh.2012.11.012)

9 Heidenreich PA, Trogdon JG, Khavjou OA, Butler J, Dracup K, Ezekowitz MD, Finkelstein EA, Hong Y, Johnston SC, Khera A, et al. Forecasting the future of cardiovascular disease in the United States: a policy statement from the American Heart Association. Circulation 2011123 933-944. (https://doi.org/10.1161/CIR.0b013e31820a55f5)

10 Regitz-Zagrosek V \& Kararigas G. Mechanistic pathways of sex differences in cardiovascular disease. Physiological Reviews 201797 1-37. (https://doi.org/10.1152/physrev.00021.2015)

11 Thaler MA, Seifert-Klauss V \& Luppa PB. The biomarker sex hormone-binding globulin - from established applications to emerging trends in clinical medicine. Best Practice and Research: Clinical Endocrinology and Metabolism 201529 749-760. (https://doi. org/10.1016/j.beem.2015.06.005)

12 Hyde Z, Norman PE, Flicker L, Hankey GJ, Almeida OP, McCaul KA, Chubb SA \& Yeap BB. Low free testosterone predicts mortality from cardiovascular disease but not other causes: the Health in Men Study. Journal of Clinical Endocrinology and Metabolism 201297 179-189. (https://doi.org/10.1210/jc.2011-1617)

13 Barrett-Connor E \& Khaw KT. Endogenous sex hormones and cardiovascular disease in men. A prospective population-based study. Circulation 198878 539-545. (https://doi.org/10.1161/01.cir.78.3.539)

14 Tivesten A, Vandenput L, Labrie F, Karlsson MK, Ljunggren O, Mellström D \& Ohlsson C. Low serum testosterone and estradiol predict mortality in elderly men. Journal of Clinical Endocrinology and Metabolism 200994 2482-2488. (https://doi.org/10.1210/jc.20082650)

15 Goodman-Gruen D \& Barrett-Connor E. A prospective study of sex hormone-binding globulin and fatal cardiovascular disease in Rancho Bernardo men and women. Journal of Clinical Endocrinology and Metabolism 199681 2999-3003. (https://doi.org/10.1210/ jcem.81.8.8768865)

16 Schaffrath G, Kische H, Gross S, Wallaschofski H, Völzke H, Dörr M, Nauck M, Keevil BG, Brabant G \& Haring R. Association of sex hormones with incident 10-year cardiovascular disease and mortality in women. Maturitas 201582 424-430. (https://doi.org/10.1016/j. maturitas.2015.08.009)

17 Wehr E, Pilz S, Boehm BO, Grammer TB, Marz W \& ObermayerPietsch B. Low free testosterone levels are associated with all-cause and cardiovascular mortality in postmenopausal diabetic women. Diabetes Care 201134 1771-1777. (https://doi.org/10.2337/dc110596)

18 Ruige JB, Mahmoud AM, De Bacquer D \& Kaufman JM. Endogenous testosterone and cardiovascular disease in healthy men: a metaanalysis. Heart 201197 870-875. (https://doi.org/10.1136/ hrt.2010.210757)

19 Araujo AB, Dixon JM, Suarez EA, Murad MH, Guey LT \& Wittert GA. Clinical review: endogenous testosterone and mortality in men: a systematic review and meta-analysis. Journal of Clinical Endocrinology and Metabolism 201196 3007-3019. (https://doi.org/10.1210/ jc.2011-1137)
20 Bianchi VE. Testosterone, myocardial function, and mortality. Heart Failure Reviews 201823 773-788. (https://doi.org/10.1007/s10741018-9721-0)

21 Muraleedharan V \& Hugh Jones TH. Testosterone and mortality. Clinical Endocrinology 201481 477-487. (https://doi.org/10.1111/ cen.12503)

22 Corona G, Rastrelli G, Monami M, Guay A, Buvat J, Sforza A Forti G, Mannucci E \& Maggi M. Hypogonadism as a risk factor for cardiovascular mortality in men: a meta-analytic study. European Journal of Endocrinology 2011165 687-701. (https://doi.org/10.1530/ EJE-11-0447)

23 Decaroli MC \& Rochira V. Aging and sex hormones in males. Virulence 20178 545-570. (https://doi.org/10.1080/21505594.2016.1 259053)

24 Barrett-Connor E \& Goodman-Gruen D. Prospective study of endogenous sex hormones and fatal cardiovascular disease in postmenopausal women. BMJ 1995311 1193-1196. (https://doi. org/10.1136/bmj.311.7014.1193)

25 Sievers C, Klotsche J, Pieper L, Schneider HJ, März W, Wittchen HU, Stalla GK \& Mantzoros C. Low testosterone levels predict all-cause mortality and cardiovascular events in women: a prospective cohort study in German primary care patients. European Journal of Endocrinology 2010163 699-708. (https://doi.org/10.1530/EJE-100307)

26 Benn M, Voss SS, Holmegard HN, Jensen GB, Tybjaerg-Hansen A \& Nordestgaard BG. Extreme concentrations of endogenous sex hormones, ischemic heart disease, and death in women. Arteriosclerosis, Thrombosis, and Vascular Biology 201535 471-477. (https://doi.org/10.1161/ATVBAHA.114.304821)

27 Handelsman DJ. Androgen physiology, pharmacology and abuse. In Endotext. Eds KR Feingold, B Anawalt, A Boyce, et al. South Dartmouth, MA, USA: MDText.com Inc, 2000. (available at: (https:// www.ncbi.nlm.nih.gov/books/NBK279000/)

28 Yeap BB, Alfonso H, Chubb SAP, Hankey GJ, Handelsman DJ, Golledge J, Almeida OP, Flicker L \& Norman PE. In older men, higher plasma testosterone or dihydrotestosterone is an independent predictor for reduced incidence of stroke but not myocardial infarction. Journal of Clinical Endocrinology and Metabolism 201499 4565-4573. (https://doi.org/10.1210/jc.2014-2664)

29 Shores MM, Biggs ML, Arnold AM, Smith NL, Longstreth WT, Kizer JR, Hirsch CH, Cappola AR \& Matsumoto AM. Testosterone, dihydrotestosterone, and incident cardiovascular disease and mortality in the cardiovascular health study. Journal of Clinical Endocrinology and Metabolism 201499 2061-2068. (https://doi. org/10.1210/jc.2013-3576)

30 Araujo AB, Kupelian V, Page ST, Handelsman DJ, Bremner WJ \& McKinlay JB. Sex steroids and all-cause and cause-specific mortality in men. Archives of Internal Medicine 2007167 1252-1260. (https:// doi.org/10.1001/archinte.167.12.1252)

31 Chan YX, Knuiman MW, Hung J, Divitini ML, Beilby JP, Handelsman DJ, Beilin J, McQuillan B \& Yeap BB. Neutral associations of testosterone, dihydrotestosterone and estradiol with fatal and non-fatal cardiovascular events, and mortality in men aged 17-97 years. Clinical Endocrinology 201685 575-582. (https://doi. org/10.1111/cen.13089)

32 Laxy M, Knoll G, Schunk M, Meisinger C, Huth C \& Holle R. Quality of diabetes care in Germany improved from 2000 to 2007 to 2014, but improvements diminished since 2007. Evidence from the population-based KORA studies. PLOS ONE 201611 e0164704. (https://doi.org/10.1371/journal.pone.0164704)

33 von Toerne C, Huth C, de Las Heras Gala T, Kronenberg F, Herder C, Koenig W, Meisinger C, Rathmann W, Waldenberger M, Roden M, et al. MASP1, THBS1, GPLD1 and ApoA-IV are novel biomarkers associated with prediabetes: the KORA F4 study. Diabetologia 201659 1882-1892. (https://doi.org/10.1007/s00125-016-4024-2) https://ec.bioscientifica.com https://doi.org/10.1530/EC-20-0080 (c) 2020 The authors Published by Bioscientifica Ltd

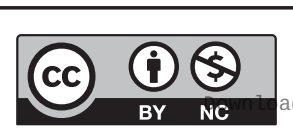

This work is licensed under a Creative Commons Attribution-NonCommercial 4.0 International License. ded from Bioscientifica.com at 04/26/2023 10:12:20AM 
34 Vermeulen A, Verdonck L \& Kaufman JM. A critical evaluation of simple methods for the estimation of free testosterone in serum. Journal of Clinical Endocrinology and Metabolism 199984 3666-3672. (https://doi.org/10.1210/jcem.84.10.6079)

35 Rosner W, Auchus RJ, Azziz R, Sluss PM \& Raff H. Position statement: utility, limitations, and pitfalls in measuring testosterone: an Endocrine Society position statement. Journal of Clinical Endocrinology and Metabolism 200792 405-413. (https://doi.org/10.1210/jc.20061864)

36 Meisinger C, Thorand B, Schneider A, Stieber J, Doring A \& Lowel H. Sex differences in risk factors for incident type 2 diabetes mellitus: the Monica Augsburg cohort study. Archives of Internal Medicine 2002 162 82-89. (https://doi.org/10.1001/archinte.162.1.82)

37 Levey AS, Stevens LA, Schmid CH, Zhang YL, Castro AF, Feldman HI, Kusek JW, Eggers P, Van Lente F, Greene T, et al. A new equation to estimate glomerular filtration rate. Annals of Internal Medicine 2009 150 604-612. (https://doi.org/10.7326/0003-4819-150-9-20090505000006)

38 Therneau TM \& Grambsch PM. Modeling survival data: extending the Cox model. In Statistics for Biology and Health. New York, NY, USA: Springer, 2000. (https://doi.org/10.1007/978-1-4757-3294-8)

39 Grambsch PM \& Therneau TM. Proportional hazards tests and diagnostics based on weighted residuals. Biometrika $1994 \mathbf{8 1}$ 515-526. (https://doi.org/10.1093/biomet/81.3.515)

40 Khaw KT, Dowsett M, Folkerd E, Bingham S, Wareham N, Luben R, Welch A \& Day N. Endogenous testosterone and mortality due to all causes, cardiovascular disease, and cancer in men: European prospective investigation into cancer in Norfolk (EPIC-Norfolk) Prospective Population Study. Circulation 2007116 2694-2701. (https://doi.org/10.1161/CIRCULATIONAHA.107.719005)

41 Lehtonen A, Huupponen R, Tuomilehto J, Lavonius S, Arve S, Isoaho H, Huhtaniemi I \& Tilvis R. Serum testosterone but not leptin predicts mortality in elderly men. Age and Ageing 200837 461-464. (https://doi.org/10.1093/ageing/afn048)

42 Hsu B, Cumming RG, Naganathan V, Blyth FM, Le Couteur DG, Hirani V, Waite LM, Seibel MJ \& Handelsman DJ. Temporal changes in androgens and estrogens are associated with all-cause and causespecific mortality in older men. Journal of Clinical Endocrinology and Metabolism 2016101 2201-2210. (https://doi.org/10.1210/ jc.2016-1025)

43 Tint AN, Hoermann R, Wong H, Ekinci EI, MacIsaac RJ, Jerums G, Zajac JD \& Grossmann M. Association of sex hormone-binding globulin and free testosterone with mortality in men with type 2 diabetes mellitus. European Journal of Endocrinology 2016174 59-68. (https://doi.org/10.1530/EJE-15-0672)

44 Kautzky-Willer A, Harreiter J \& Pacini G. Sex and gender differences in risk, pathophysiology and complications of type 2 diabetes mellitus. Endocrine Reviews 201637 278-316. (https://doi. org/10.1210/er.2015-1137)

45 Joyce KE, Biggs ML, Djousse L, Ix JH, Kizer JR, Siscovick DS, Shores MM, Matsumoto AM \& Mukamal KJ. Testosterone, dihydrotestosterone, sex hormone-binding globulin, and incident diabetes among older men: the cardiovascular health study. Journal of Clinical Endocrinology and Metabolism 2017102 33-39. (https://doi. org/10.1210/jc.2016-2623)

46 Jaruvongvanich V, Sanguankeo A, Riangwiwat T \& Upala S. Testosterone, sex hormone-binding globulin and nonalcoholic fatty liver disease: a systematic review and meta-analysis. Annals of Hepatology 201716 382-394. (https://doi. org/10.5604/16652681.1235481)

47 Burger HG. Androgen production in women. Fertility and Sterility 200277 (Supplement 4) S3-S5. (https://doi.org/10.1016/s00150282(02)02985-0)

48 Menke A, Guallar E, Rohrmann S, Nelson WG, Rifai N, Kanarek N, Feinleib M, Michos ED, Dobs A \& Platz EA. Sex steroid hormone concentrations and risk of death in US men. American Journal of Epidemiology 2010171 583-592. (https://doi.org/10.1093/aje/ kwp415)

49 Kalyani RR, Gavini S \& Dobs AS. Male hypogonadism in systemic disease. Endocrinology and Metabolism Clinics of North America 2007 36 333-348. (https://doi.org/10.1016/j.ecl.2007.03.014)

50 Kiddy DS, Hamilton-Fairley D, Seppala M, Koistinen R, James VH, Reed MJ \& Franks S. Diet-induced changes in sex hormone binding globulin and free testosterone in women with normal or polycystic ovaries: correlation with serum insulin and insulin-like growth factor-I. Clinical Endocrinology 198931 757-763. (https://doi. org/10.1111/j.1365-2265.1989.tb01297.x)

51 Vandenput L, Mellström D, Kindmark A, Johansson H, Lorentzon M, Leung J, Redlund-Johnell I, Rosengren BE, Karlsson MK, Wang YX, et al. High serum SHBG predicts incident vertebral fractures in elderly men. Journal of Bone and Mineral Research 201631 683-689. (https:// doi.org/10.1002/jbmr.2718)

52 Damassa DA, Lin TM, Sonnenschein C \& Soto AM. Biological effects of sex hormone-binding globulin on androgen-induced proliferation and androgen metabolism in LNCaP prostate cells. Endocrinology 1991129 75-84. (https://doi.org/10.1210/endo-129-1-75)

53 Rosner W, Hryb DJ, Kahn SM, Nakhla AM \& Romas NA. Interactions of sex hormone-binding globulin with target cells. Molecular and Cellular Endocrinology 2010316 79-85. (https://doi.org/10.1016/j. mce.2009.08.009)

54 Hryb DJ, Khan MS, Romas NA \& Rosner W. The control of the interaction of sex hormone-binding globulin with its receptor by steroid hormones. Journal of Biological Chemistry 1990265 6048-6054.

55 Liu PY, Death AK \& Handelsman DJ. Androgens and cardiovascular disease. Endocrine Reviews 200324 313-340. (https://doi.org/10.1210/ er.2003-0005)

56 Brambilla DJ, Matsumoto AM, Araujo AB \& McKinlay JB. The effect of diurnal variation on clinical measurement of serum testosterone and other sex hormone levels in men. Journal of Clinical Endocrinology and Metabolism 200994 907-913. (https://doi.org/10.1210/ jc.2008-1902)

57 Hackbarth JS, Hoyne JB, Grebe SK \& Singh RJ. Accuracy of calculated free testosterone differs between equations and depends on gender and SHBG concentration. Steroids 201176 48-55. (https://doi. org/10.1016/j.steroids.2010.08.008)

Received in final form 21 February 2020

Accepted 13 March 2020

Accepted Manuscript published online 13 March 2020 https://ec.bioscientifica.com https://doi.org/10.1530/EC-20-0080 (c) 2020 The authors Published by Bioscientifica Ltd

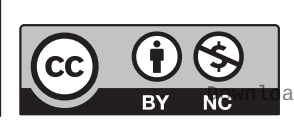

This work is licensed under a Creative Commons Attribution-NonCommercial 4.0 International License. ded from Bioscientifica com at 04/26/2023 10:12:20AM 